\title{
Multiple gastrointestinal melanoma causing small bowel intussusception
}

\author{
Jian Yang Eng ${ }^{1}$, Salehah Tahkin ${ }^{1}$, Huzairi Yaacob ${ }^{1}$, Nor Hayati Yunus², Ahmad Shan Wani Mohamed Sidek ${ }^{1}$, \\ Michael Pak-Kai Wong ${ }^{3}$ \\ ${ }^{1}$ Department of Surgery, Hospital Raja Perempuan Zainab II, Kota Bharu, Malaysia \\ ${ }^{2}$ Department of Pathology, Hospital Raja Perempuan Zainab II, Kota Bharu, Malaysia \\ ${ }^{3}$ School of Medical Sciences, Hospital Universiti Sains Malaysia, Kota Bharu, Malaysia
}

\begin{abstract}
A 61-year-old gentleman presented with small bowel intussusception from small bowel melanoma intussusceptum. He complains of intermittent abdominal distension but no history of intestinal obstruction. Apart from this, he was also symptomatic anemia which required repeated transfusion for the past few months. The contrast-enhanced computed tomography of the abdomen shows an omental mass with small bowel intussusception. He then underwent an exploratory laparotomy with segmental resection of the affected segment. Histopathological examination confirmed primary gastrointestinal melanoma. Multiple small bowel malignant melanoma is a rare disease. It remains a controversial diagnosis as it may be a primary or metastasis from an unidentified or regressed primary cutaneous melanoma. Prompt surgical intervention enables us to obtain tissue diagnosis, prevent complete intestinal obstruction and strategize the goals of treatment for the patient.
\end{abstract}

Keywords: Melanoma; Intussusception; Intestinal obstruction; Acute abdomen

\section{INTRODUCTION}

Malignant melanoma is a common malignancy of the skin. It frequently metastasizes the gastrointestinal (GI) tract, with up to $60 \%$ of cases presented as advanced metastatic melanoma $[1,2]$. Primary malignant melanoma of small bowel is a rare clinical entity. It remains a controversial diagnosis as it could be metastasized from an unidentified or regressed primary cutaneous melanoma $[3,4]$. The clinical presentation was not specific, vague abdominal pain, unexplained weight loss, anemia, and GI bleeding, leading to late diagnosis. Intussusception from melanoma as the pathological lead point poses a challenge to diagnose, especially when it is an uncomplicated, asymptomatic case [5]. The inciden-

Received: Feb 23, 2021 - Revised: Apr 4, 2021 - Accepted: Apr 21, 2021 Correspondence to: Michael Pak-Kai Wong, MBChB, M.Med Surgery Department of Surgery, School of Medical Sciences, Hospital Universiti Sains Malaysia, Kubang Kerian, 15200 Kota Bharu, Kelantan, Malaysia Email: michaelpkwong@usm.my ORCID: https://orcid.org/0000-0001-9137-3096

(C) 2023 The Korean Society of Coloproctology

This is an open-access article distributed under the terms of the Creative Commons Attribution NonCommercial License (https://creativecommons.org/licenses/by-nc/4.0) which permits unrestricted noncommercial use, distribution, and reproduction in any medium, provided the original work is properly cited. tal finding on imaging would often require surgical resection to confirm the diagnosis of intestinal melanoma [1, 5]. GI melanoma, likewise any mucosal melanoma, are more aggressive than cutaneous melanoma with a median survival of about 4 to 6 months and a 5-year overall survival rate of less than 10\% [6]. Despite the poor prognosis, operative management remains the mainstay of treatment in small bowel melanoma. There was little evidence on the benefit of chemotherapy $[6,7]$. Though there was a report on dacarbazine, many did not survive long enough to initiate the palliative treatment [8].

We are reporting a case of 61-year-old man with multiple small bowel melanoma, presented with unexplained anemia and uncomplicated, asymptomatic intussusception.

Written informed consent was obtained for publication of this case report and accompanying images.

\section{CASE REPORT}

A 61-year-old man presented to a district general hospital with multiple episodes of symptomatic anemia requiring transfusion. He has been generally unwell for the past 1 month with predominant complaints of lethargy and dizziness. He did not complain of any alteration of bowel habit and GI bleeding. He was presented 


\section{Coloproctology $\quad$ Jian Yang Eng, et al.}
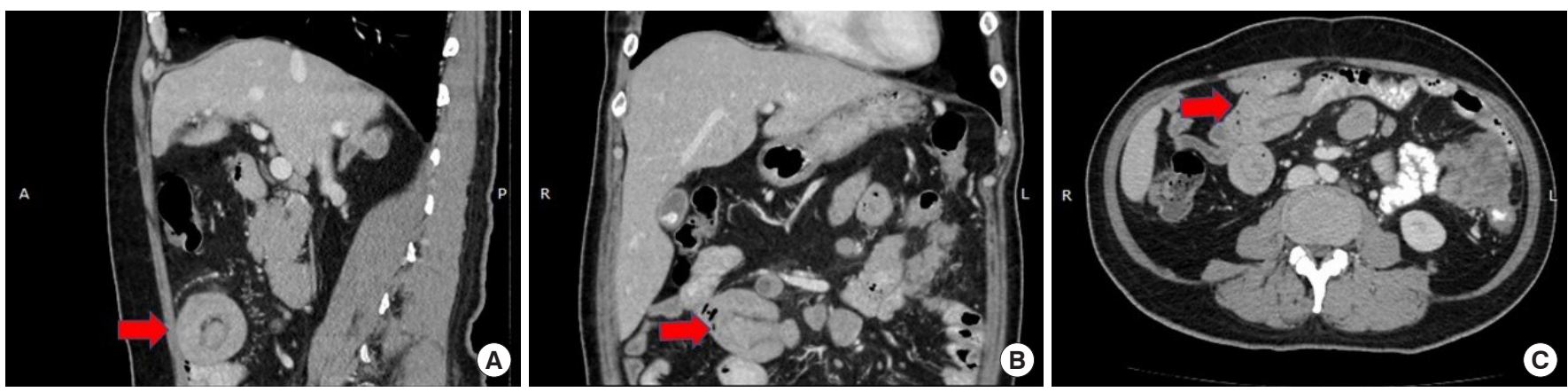

Fig. 1. Multiplanar view of the contrast-enhanced computed tomography of the abdomen indicating the small bowel intussusception at right lumbar region (red arrow). (A) Sagittal view. (B) Coronal view. (C) Axial view. A, anterior; P, posterior; R, right; L, left.
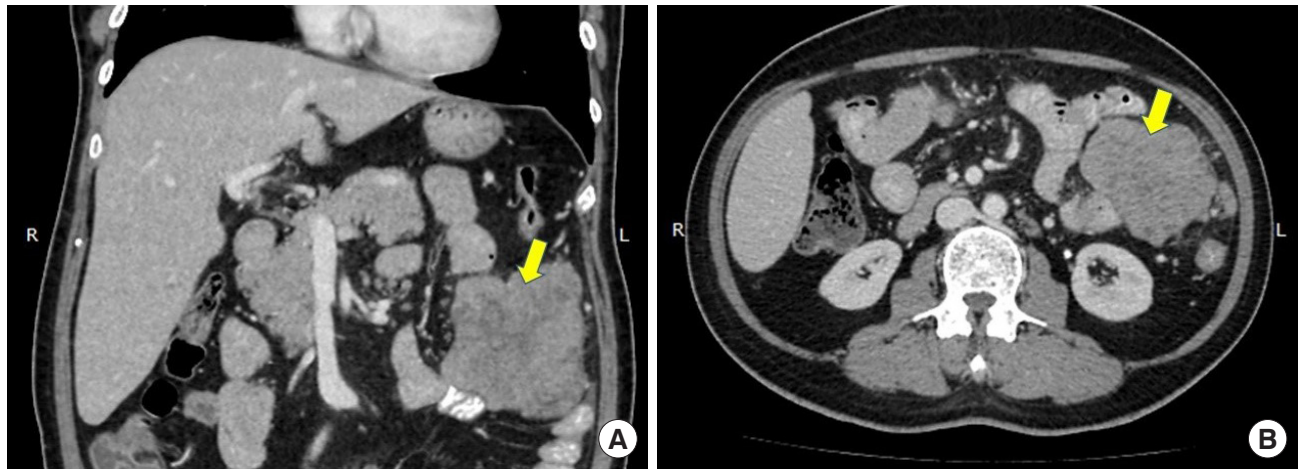

Fig. 2. Multiplanar view of the contrast-enhanced computed tomography of the abdomen showing the heterogeneous soft tissue mass at left lumbar region suggests an omental mass (yellow arrow). (A) Coronal view. (B) Axial view. R, right; L, left.

to us with hemoglobin of $4.9 \mathrm{~g} / \mathrm{dL}$ and was given blood transfusion due to symptomatic anemia. His full blood pictured suggestive of iron deficiency anemia

On examination, he appeared pale and his vital signs were stable. Abdominal examination revealed a non-tender mass at the left lumbar region measuring about $7 \times 7 \mathrm{~cm}$. There were multiple lymphadenopathies of various sizes located at the left supraclavicular and bilateral inguinal region.

The contrast-enhanced computed tomography of the abdomen was performed and confirmed the diagnosis of small bowel intussusception (Fig. 1) and another lobulated heterogeneous enhancing soft tissue mass at the left lumbar region (Fig. 2). The initial diagnosis was GI lymphoma causing small bowel intussusception.

An emergency laparotomy was performed after informed consent from the patient as persistent anemia may be resulting from occult GI tumor bleeding and the intussusception may progress to obstruction soon. Intraoperatively, there was a large omental tumor, near the distal transverse colon, measuring $11 \times 9 \mathrm{~cm}$; the tumor was hyperpigmented irregular outer surface. There was a clear plane between the tumor and the adjacent colon (Fig. 3). Multiple jejunojejunal intussusception sites were identified with intraluminal hyperpigmented polypoidal lesions of varying sizes felt throughout the jejunum. There were also multiple mesentery
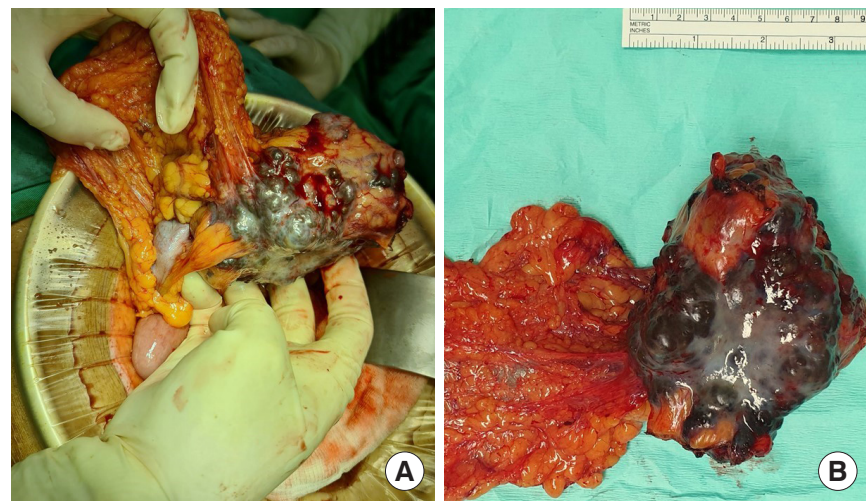

Fig. 3. Hyperpigmented omental tumor. (A) The intraoperative view. (B) The size of the removed hyperpigmented omental tumor.

lymphadenopathies with hyperpigmentation noted (Fig. 4). This segment was resected in view of potential obstruction with a total length of jejunum $80 \mathrm{~cm}$ resected with primary end-to-end anastomosis.

Histology of the omental tumor showed infiltration of malignant cells with intracytoplasmic pigments and intermingle extracellular pigmentation. The cells had enlarged nuclei, abundant cy- 

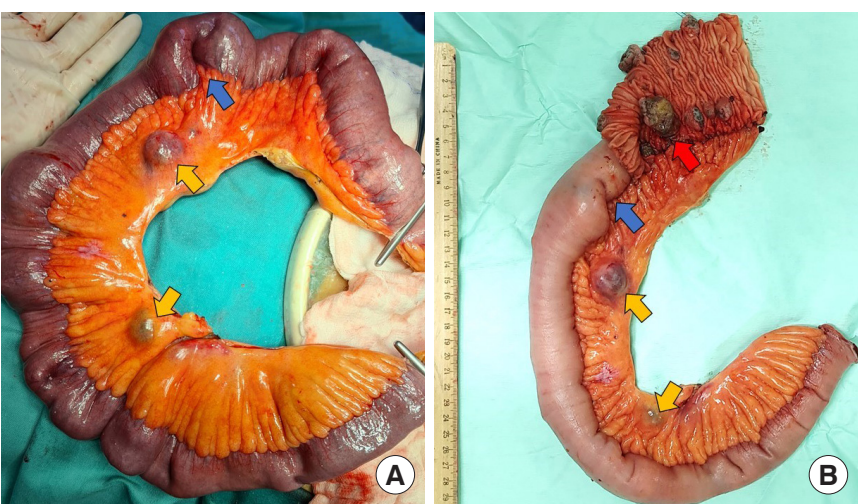

Fig. 4. Segmental resection of jejunum. Intraluminal hyperpigmented polyps (red arrow), intussusception point (blue arrows), and multiple hyperpigmented mesentery lymphadenopathies (yellow arrows). (A) The intraoperative view. (B) The measurement of the affected resected segment of jejunum.

toplasm and show brisk mitotic activity. The small bowel polypoidal lesions had similar histological features, with infiltration of malignant cells to the surface of mucosal causing surface ulceration. Immunohistochemical staining of melan-A, S-100, and human melanoma black 45 was strongly positive, suggesting a GI melanoma (Fig. 5). Retrospectively, we performed a thorough physical examination to look for cutaneous or ocular melanoma on him but in vain. He had an uneventful postoperative recovery and was discharged 4 days after his operation.

\section{DISCUSSION}

Noncutaneous melanoma occurs very rarely. Cutaneous melanoma is commonly seen with metastasis to the GI tract, due to its rich vascular supply $[1,7]$. It contributes to $50 \%$ to $70 \%$ of small bowel secondary malignancy [3]. In contrast, primary malignant melanoma of the small bowel is a rare entity. Differentiating between primary and secondary small bowel melanoma remained debatable because of the possibility of small bowel secondary deposits from an indolent or regressed primary cutaneous melanoma [3]. Hypothetically, small bowel mucosa does not usually contain melanocytes; hence, should not be the primary site of the melanoma. The etiology of primary melanoma in the small bowel is unclear and speculative. The 2 favorable hypotheses were the migration of melanocytes into the GI tract as observed through the occasional formation of benign melanosis in esophageal mucosa and the more widely accepted hypothesis of amine precursors uptake and decarboxylation (APUD) theory [4]. It was postulated that neural crest cells migrate from the umbilical mesenteric canal into the GI tract, which later differentiate into APUD cells. These cells undergo neoplastic transformation to form melanoma which may explain the commonest site of GI melanoma being at ileum, the distal end of umbilical mesenteric canal $[1,3,4,6]$.
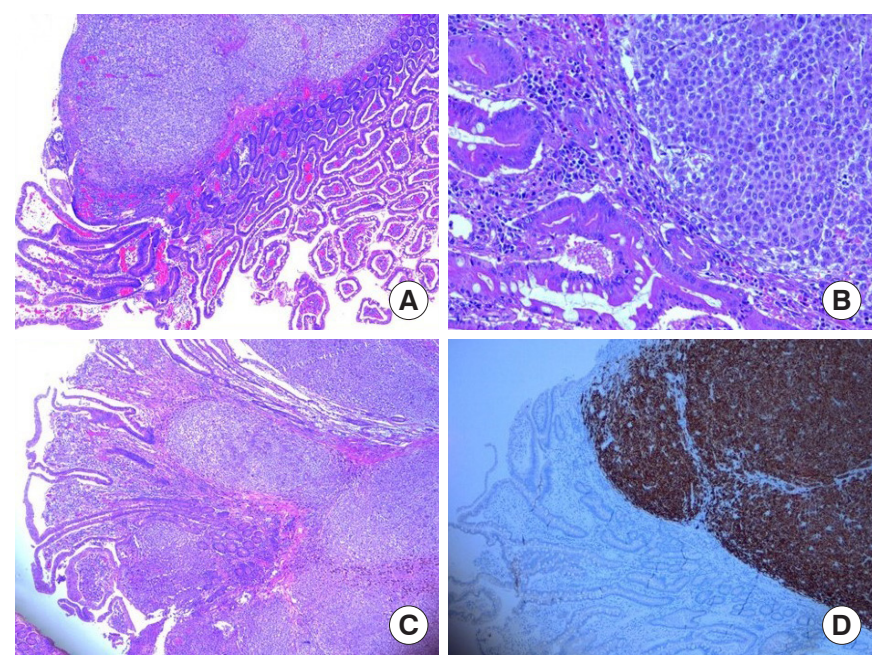

Fig. 5. (A) Small bowel polyps showing malignant cells at the lamina propria and submucosa $(\mathrm{H} \& \mathrm{E}, \times 4)$. (B) The malignant cells are arranged in diffuse sheets with presence of intracytoplasmic and extracellular pigments $(H \& E, \times 20)$. (C) Small bowel polyps showing malignant cells at the lamina propria and submucosa $(\mathrm{H} \& \mathrm{E}, \times 4)$. (D) These cells are strongly positive for melan-A immunohistochemistry stain $(\times 4)$.

The diagnosis of small bowel melanoma is challenging due to its vague clinical features and limited endoscopic access [4]. The common symptoms may include GI bleeding, abdominal pain, unintentional drastic weight loss, and anemia. Acute presentations of intussusception and bowel perforation are rare. Similarly, in our patient, his initial presentation was symptomatic anemia with no obvious GI bleeding. He had no symptom of intestinal obstruction related to the intussusception.

The criteria to diagnose primary small bowel melanoma are single solitary tumor in the small bowel mucosa, presence of other intramucosal melanocytic lesions in surrounding intestinal epithelium, and absence of concurrent or regressed cutaneous melanoma or another atypical melanocytic lesion $[1,7]$. Multifocal lesions were usually suggestive of metastasis and only about $9 \%$ of multifocal melanomas have no extraintestinal melanocytic lesion identified as the primary lesion [7].

In our case, there were multiple intraluminal melanocytic lesions were identified in jejunum, causing multiple sites of jejunojenunal intussusception. There were numerous mesenteric lymphadenopathies showing metastatic melanoma. The presence of a large irregular hyperpigmented omental tumor confirmed metastatic melanoma. Despite its multifocality and evidence of metastases, there was no extraintestinal melanocytic lesion identified. Therefore, this was a rare presentation of multifocal jejunal melanoma with metastases.

Small bowel melanoma is aggressive, and with metastasis, its prognosis is worse than the cutaneous or extraintestinal melanomas. The medial survival time reported is about 6 months with 5 
years overall survival of less than $10 \%[5,6]$. The aggressive tumor biology with poor prognosis making many reserved for the benefit from operative exploration in the asymptomatic patient. It has been reported that the incidental intussusception often resolves spontaneously on follow-up imaging, and these patients can be managed expectantly [5]. However, follow-up on a case series demonstrated that $50 \%$ would eventually require complicated intussusception surgery. Therefore, a low threshold for operative intervention as a palliative or preventive measure for incidental intussusception in patients with melanoma to prevent potential complications such as obstruction and GI bleeding $[5,6]$.

In our case, exploration and surgical resection prevented the complications as such and to obtain a histopathological diagnosis for further planning of treatment.

Adjuvant therapeutic modalities in small bowel melanoma are limited to palliative goal with dacarbazine $[1,8]$. Early detection and surgical resection still provide the best long-term disease-free survival [7]. In the era of advancement of targeted therapy and immunotherapy, the treatment of metastatic melanoma has been revolutionized with immune checkpoint inhibitors and targeted BRAF/MEK inhibitors [9]. The landmark trial CheckMate-067 explore the efficacy of ipilimumab/nivolumab combination compared with monotherapy for advanced melanoma and concluded the superior results in the combination group in improving the median overall survival from 37 months to more than 60 months [9].

Malignant melanoma of the small bowel is a rare entity that may present late as the complication of intussusception, perforation, and GI bleeding. Early detection and surgical resection provide better long-term disease-free survival for curative and palliative goals.

\section{CONFLICT OF INTEREST}

No potential conflict of interest relevant to this article was reported.

\section{FUNDING}

None.

\section{ACKNOWLEDGMENTS}

We would like to express gratitude all the medical officers from Green Team (Colorectal Unit), Hospital Raja Perempuan Zainab II (Kota Bharu, Malaysia) for their relentless effort during the care of this patient.

\section{REFERENCES}

1. Sinagra E, Sciumè C. Ileal melanoma, a rare cause of small bowel obstruction: report of a case, and short literature review. Curr Radiopharm 2020;13:56-62.

2. Silva S, Tenreiro N, Melo A, Lage J, Moreira H, Próspero F, et al. Metastatic melanoma: an unusual cause of gastrointestinal bleeding and intussusception-a case report. Int J Surg Case Rep 2018; 53:144-6.

3. Olatoke SA, Agodirin SO, Adenuga AT, Lawal BO, Ibrahim KO, Folaranmi OO. Primary jejunal melanoma as a cause of adult intussusception: a case report and review of literature. Pan Afr Med J 2019;33:214.

4. Simons M, Ferreira J, Meunier R, Moss S. Primary versus metastatic gastrointestinal melanoma: a rare case and review of current literature. Case Rep Gastrointest Med 2016;2016:2306180.

5. Perez MC, Sun J, Farley C, Han D, Sun AH, Narayan D, et al. Management of intussusception in patients with melanoma. J Surg Oncol 2019;119:897-902.

6. Vrable A, Chang R. Malignant melanoma of the small bowel presenting with intussusception in a woman: a case report. Melanoma Manag 2017;4:99-104.

7. Kouvaras S, Rokkas T, Goga H, Gakiopoulou H, Arapantoni P, Haliotis G, et al. Multifocal gastrointestinal melanoma. J Gastrointestin Liver Dis 2019;28:237-40.

8. Mohamad H, Rahman WF, Zain WZ, Zakaria AD, Aziz SH, Wong MP. Aggressive pigmented anal melanoma. Surg Chron 2018;23:127-9.

9. Kohoutova D, Worku D, Aziz H, Teare J, Weir J, Larkin J. Malignant melanoma of the gastrointestinal tract: symptoms, diagnosis, and current treatment options. Cells 2021;10:327. 